essentials 
essentials liefern aktuelles Wissen in konzentrierter Form. Die Essenz dessen, worauf es als „State-of-the-Art“" in der gegenwärtigen Fachdiskussion oder in der Praxis ankommt. essentials informieren schnell, unkompliziert und verständlich

- als Einführung in ein aktuelles Thema aus Ihrem Fachgebiet

- als Einstieg in ein für Sie noch unbekanntes Themenfeld

- als Einblick, um zum Thema mitreden zu können

Die Bücher in elektronischer und gedruckter Form bringen das Expertenwissen von Springer-Fachautoren kompakt zur Darstellung. Sie sind besonders für die Nutzung als eBook auf Tablet-PCs, eBook-Readern und Smartphones geeignet. essentials: Wissensbausteine aus den Wirtschafts-, Sozial- und Geisteswissenschaften, aus Technik und Naturwissenschaften sowie aus Medizin, Psychologie und Gesundheitsberufen. Von renommierten Autoren aller Springer-Verlagsmarken.

Weitere Bände in dieser Reihe http://www.springer.com/series/13088 
Josef Wiemeyer

\section{Serious Games für \\ die Gesundheit}

Anwendung in der Prävention und Rehabilitation im Überblick

Springer 
Prof. Dr. rer. medic. Josef Wiemeyer

Technische Universität Darmstadt

Darmstadt, Deutschland

ISSN 2197-6708

essentials

ISBN 978-3-658-15471-4

ISSN 2197-6716 (electronic)

DOI 10.1007/978-3-658-15472-1

ISBN 978-3-658-15472-1 (eBook)

Die Deutsche Nationalbibliothek verzeichnet diese Publikation in der Deutschen Nationalbibliografie; detaillierte bibliografische Daten sind im Internet über http://dnb.d-nb.de abrufbar.

(C) Springer Fachmedien Wiesbaden 2016

Das Werk einschließlich aller seiner Teile ist urheberrechtlich geschützt. Jede Verwertung, die nicht ausdrücklich vom Urheberrechtsgesetz zugelassen ist, bedarf der vorherigen Zustimmung des Verlags. Das gilt insbesondere für Vervielfältigungen, Bearbeitungen, Übersetzungen, Mikroverfilmungen und die Einspeicherung und Verarbeitung in elektronischen Systemen.

Die Wiedergabe von Gebrauchsnamen, Handelsnamen, Warenbezeichnungen usw. in diesem Werk berechtigt auch ohne besondere Kennzeichnung nicht zu der Annahme, dass solche Namen im Sinne der Warenzeichen- und Markenschutz-Gesetzgebung als frei zu betrachten wären und daher von jedermann benutzt werden dürften.

Der Verlag, die Autoren und die Herausgeber gehen davon aus, dass die Angaben und Informationen in diesem Werk zum Zeitpunkt der Veröffentlichung vollständig und korrekt sind. Weder der Verlag noch die Autoren oder die Herausgeber übernehmen, ausdrücklich oder implizit, Gewähr für den Inhalt des Werkes, etwaige Fehler oder Äußerungen.

Gedruckt auf säurefreiem und chlorfrei gebleichtem Papier

Springer ist Teil von Springer Nature

Die eingetragene Gesellschaft ist Springer Fachmedien Wiesbaden GmbH

Die Anschrift der Gesellschaft ist: Abraham-Lincoln-Str. 46, 65189 Wiesbaden, Germany 


\section{Was Sie in diesem essential finden können}

- Eine Einführung in die Komplexität des Gesundheitskonzeptes einschließlich Prävention und Rehabilitation

- Eine Einführung in Serious Games und ihre Doppelmission

- Einen Überblick über die Einsatzmöglichkeiten von Serious Games in verschiedenen Bereichen der Prävention und Rehabilitation

- Einen kritischen Überblick über wissenschaftliche Untersuchungen zum Einsatz von Serious Games für die Gesundheit

- Praktische Empfehlungen zum Einsatz von Serious Games im Gesundheitsbereich 


\section{Vorwort}

Dieses Werk basiert auf zahlreichen Kooperationen des Autors mit IT-Experten sowie Experten in verschiedenen Anwendungsfeldern von Serious Games. Es wurde mit der Intention geschrieben, allen an dieser Thematik Interessierten einen konzisen Ein- und Überblick zu Serious Games für die Gesundheit zu geben. Der Autor möchte sich herzlich für die von den Projektpartnern gewährte Unterstützung bedanken. Schließlich gilt ein besonderer Dank dem Springer-Verlag, der die Entstehung dieses Werkes engagiert, konstruktiv und mit der nötigen Behutsamkeit begleitet hat.

Der Autor verbindet mit diesem Werk die Hoffnung, dass digitale Spiele in ihrer potenziellen Bedeutung für die Gesundheit erkannt werden und einen geeigneten Platz im Arsenal der Gesundheitsinterventionen finden.

Darmstadt, Deutschland

Josef Wiemeyer 


\section{Inhaltsverzeichnis}

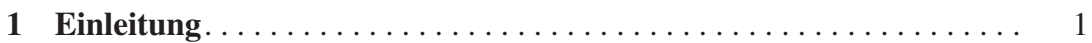

2 Gesundheit - Begriff, Modelle und Interventionen ............ 5

2.1 Gesundheit - Inhalt und Struktur eines

komplexen Begriffs ..................... 5

2.2 Gesundheitsmodelle........................ 6

2.3 Gesundheitsinterventionen - Arten, Bereiche und Ziele . . . . . . . . 7

2.4 Rahmenbedingungen und gesetzliche Bestimmungen........ 10

3 Spielen, digitale Spiele, Serious Games und

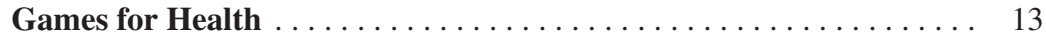

3.1 Spielen als fundamentale menschliche Tätigkeit ........... 13

3.2 Digitale Spiele - Arten und Wirkungen . . . . . . . . . . . . . 14

3.3 Serious Games - Definition, Arten und

Anwendungsbereiche...................... 17

3.4 Games for Health - Serious Games für die Gesundheit . . . . . . . 19

4 Serious Games als Präventionsmittel $\ldots \ldots \ldots \ldots \ldots \ldots \ldots \ldots 23$

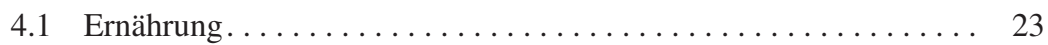

4.2 Körperliche Aktivität und Energieverbrauch ............. 25

4.3 Allgemeine Überblicksarbeiten . . . . . . . . . . . . . . . . . . . 29

4.4 Praktische Empfehlungen zum Einsatz von

DG4H in der Prävention. . . . . . . . . . . . . . . . . 31 
5 Serious Games als Rehabilitations- bzw.

Therapiemittel........................... 33

$5.1 \quad$ Krebs-Therapie........................... 33

5.2 Neurologische Erkrankungen................... 35

5.3 Überblicksarbeiten ......................... 38

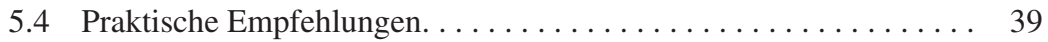

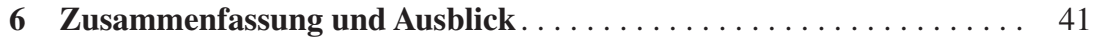

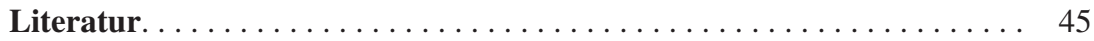

